\title{
Acute Neurobrucellosis: Syndrome of Inappropriate Antidiuretic Hormone Secretion and Parkinsonism Presentation
}

\author{
Mohammad Yasin ${ }^{1, *} ;$ Amir Hossein Moghtader Mojdehi ${ }^{1}$ \\ ${ }^{1}$ Department of Infectious Diseases, Labbafi Nezhad Hospital, Tehran, IR Iran \\ ${ }^{*}$ Corresponding author: Mohammad Yasin, Department of Infectious Diseases, Labbafi Nezhad Hospital, Pasdaran St., Tehran, IR Iran. Tel:+98-2122549011, Fax:+98-2122549039, E-mail: \\ mohammadyassin@ymail.com
}

Received: April 6, 2014; Accepted: May 15, 2014

\begin{abstract}
Introduction: Brucellosis is a zoonotic infection with high distribution all over the world and has many clinical manifestations, one of which is neurobrucellosis (NB).

Case Presentation: Our case was a 78-year-old man who presented acute decreased level of consciousness, acute parkinsonism signs and hyponatremia due to syndrome of inappropriate antidiuretic hormone secretion (SIADH), which was caused by brucellosis.

Discussion: Neurobrucellosis is a rare presentation of brucellosis and should be considered when neurological problems cannot be explained by other etiology especially in endemic areas.
\end{abstract}

Keywords:Brucellosis; Neurobrucellosis; Inappropriate ADH Syndrome; Parkinsonism

\section{Introduction}

Brucellosis is a zoonotic infection, which is considered as one of the most widely spread animal-human infections, worldwide (1). Brucellosis can be transmitted to human hosts through numerous pathways, yet the consumption of unpasteurized dairy products is still the most common way (2). Even though the disease can be found all over the world, some areas are considered as high risk regions for brucellosis, such as the Middle East, South and Central America, and Mediterranean Basin (3). In general, Brucella species infect the reticuloendothelial system, yet they can infect any organ of the body, which can result in serious complications with poor treatment and misdiagnosis, especially in endemic areas (4). Neurobrucellosis is found in less than $5 \%$ of brucellosis cases and has various clinical presentations in both central and peripheral nervous system; meningoencephalitis and aseptic meningitis are considered as the most common forms of nervous system involvement so far (5). Neurobrucellosis should be considered when neurological problems cannot be explained by other etiology especially in endemic areas (6).

\section{Case Presentation}

A retired 78-year-old man who had been healthy was admitted to our unit as he had experienced a few hours of decreased consciousness. His problems started with a dull headache in the occipital region four days earlier accompanied with nausea, vomiting, anorexia, bradykinesia, restless tremor and rigidity. He referred to the clinic after three days, where a head CT scan and MRI was obtained, which revealed no structural or anatomic abnormalities; he developed a sudden fever 12 hours after admission. Except for tamsulosin use he wasn't under any kind of prescription and use of illicit drugs. The only suspicious point in his history was regular use of unpasteurized dairy products for many years. On physical examination he was febrile with axillary temperature of $38.6^{\circ} \mathrm{C}$, pulse rate of $114 \mathrm{beat} / \mathrm{min}$ and respiratory rate of $16 / \mathrm{min}$. The rest of the physical examination was normal, yet the neurological examination revealed an old man with a semi-consciousness state, without nuchal rigidity and papilledema, and both Kernig's and Brudzinski's signs were negative. The patient's lab tests were normal except for mild anemia and hyponatremia ( $\mathrm{Na}=125$ $\mathrm{mEq} / \mathrm{L})$; liver function tests were slightly elevated, erythrocyte sedimentation rate (ESR) was $10 \mathrm{~mm} / \mathrm{h}$, C-reactive protein [CRP] was $5 \mathrm{mg} / \mathrm{dL}$. Rapid plasma regain (RPR), HIV, and viral hepatitis serology tests were negative. Even with appropriate treatment with $3 \%$ hypertonic saline, the patient was still hyponatremic, and his sodium levels was between $125 \mathrm{mEq} / \mathrm{L}$ and $129 \mathrm{mEq} / \mathrm{L}$. The patient received intravenous empirical treatment for meningitis with $2 \mathrm{~g}$ ceftriaxone, $1 \mathrm{~g}$ vancomycin, and $2 \mathrm{~g}$ ampicillin, plus a single dose of $700 \mathrm{mg}$ acyclovir. The second brain CT had normal results, cerebrospinal Fluid (CSF) samples were collected and revealed high protein (150 mg/ $\mathrm{dL}$ ), with lymphocytic pleocytosis $(\mathrm{WBC}=65$ cell $/ \mu \mathrm{L}, 91 \%$ lymphocyte) and low glucose $(20 \mathrm{mg} / \mathrm{dL})$. The PCR of CSF 
was negative for mycobacterium tuberculosis and HSV12. Serum agglutination tests were positive; Wright 1/640 and $2 \mathrm{ME} 1 / 40$. The brucellosis antibody test was positive for IgG $(\operatorname{IgG}=165.4 \mathrm{Ru} / \mathrm{mL})$ and CSF-PCR was positive for brucellosis.

The patient's treatment was switched to doxycycline $200 \mathrm{mg} / \mathrm{q} 12 \mathrm{~h}$, oral rifampin $600 \mathrm{mg} / \mathrm{d}$ and intravenous ceftriaxone $2 \mathrm{~g} / \mathrm{q} 12 \mathrm{~h}$ for 2 weeks. After this treatment all of the patient's symptoms subsided even his uncontrolled hyponatremia and parkinsonism signs and he gained back his health. After discharge, the ceftriaxone was changed to trimethoprim/sulfamethoxazole (160/800), 2 tablets/q12h. The three-antibiotic-treatment was continued for 12 weeks with weekly follow ups.

\section{Discussion}

Brucella species are gram-negative, aerobic, encapsulated coccobacilli, which include $B$. melitensis, $B$. abortus, $B$. canis and $B$. suis. These species can infect humans (1). The first Brucella stain isolated in Iran was B. abortus, which was isolated from a bovine fetus, during the year 1944 (7). Besides, in 1950, the first B. melitensis was isolated (8). These two species are the only species isolated in Iran with B. melitensis having greater prevalence (5). Brucellosis has high incidence in Iran with a rate of about 0.73-141.6 per 100000 individuals, per year $(9,10)$. The main sources of infection are ingestion of contaminated dairy products, direct contact with infected animals, conjunctival inoculation and inhalation of contaminated aerogels (10). There are various risk factors associated with brucellosis, which can be modified by education programs, these include, having an infected individual in the household, and animal's death or abortion in family holding and raising animals also considered to be a risk factor related to animals. Educated individuals seem to have a protective factor against brucellosis (11). Neurobrucellosis is a rare presentation of brucellosis which can occur by direct or indirect mechanisms (12). Neurobrucellosis has many signs and symptoms including, headache, fever, sweats, weight loss, meningeal irritation signs, confusion, hepatosplenomegaly, convulsion disorder, dysarthria and diplopia (13). The most common clinical manifestation of NB is aseptic meningitis or meningoencephalitis $(5,9)$, but it can also manifest as polyradiculoneuritis, Guillain-Barre syndrome, brain abscess (14) and Parkinson's disease (15). Theoretically, any central nervous disorder can lead to antidiuretic hormone release which leads to syndrome of inappropriate antidiuretic hormone secretion (SIADH) and hyponatremia (16).

NB diagnosis is done by isolating the Brucella bacteria from blood or CSF which is considered as the gold standard, but cultures are negative in $>50 \%$ of the cases $(9$, 15). Cerebrospinal fluid analysis usually reveals lymphocytic pleocytosis, high protein levels and hypoglycorrhachia, except for cerebellar syndrome, which only results in high protein level $(9,16)$. Agglutination tests such as
Coombs test and Rose-Bengal test are used for diagnosing $\mathrm{NB}$, but their sensitivity is less than enzyme-linked immunosorbent assay (ELISA); real time PCR assay is a powerful tool for detecting and diagnosing NB (17).

Neurobrucellosis treatment includes the following medications, doxycycline, rifampicin, ceftriaxone, trimethoprim-sulfamethaxazol, streptomycin and ciprofloxacin, which have been found to have high effectiveness against NB (18). Treatment duration depends on the patient's condition; the duration of treatment is 24 weeks (19). Even for our patient who was an elderly with chronic use of unpasteurized dairy products, NB was without systemic involvement and the treatment was only for 12 weeks due to rapid response to the treatment. The prognosis is favorable, with mortality rate being less than $5.5 \%$. However, there are reports of permanent bilateral hearing loss and myelopathy (20). Regarding our patient, even with presentation of acute brucellosis as NB and symptoms of SIADH and Parkinsonism, he regained all of his functions as before the illness.

Neurobrucellosis is a rare presentation of brucellosis and has a variety of symptoms. This disease should be considered when neurological problems can't be explained by other etiology or when patients do not respond to treatments, especially in endemic areas.

\section{Acknowledgements}

We thank all the nursing staff and the personnel of Gholhak laboratory for their precious helps.

\section{References}

1. Corbel MJ. Brucellosis: an overview. Emerg Infect Dis. 1997; 3(2):213-21.

2. Strannegard IL, Araj GF, Fattah HA. Neurobrucellosis in an eightyear-old child. Ann Trop Paediatr. 1985;5(4):191-4.

3. Centers for Disease Control and Prevention. Brucellosis. 2009. Available at: www.cdc.gov/ncidod/dbmd/diseaseinfo/brucellosis_g html\#howcommon

4. Colmenero JD, Reguera JM, Martos F, Sanchez-De-Mora D, Delgado $\mathrm{M}$, Causse $\mathrm{M}$, et al. Complications associated with Brucella melitensis infection: a study of 530 cases. Medicine (Baltimore). 1996;75(4):195-211.

5. Zowghi E, Ebadi A, Yarahmadi M. Isolation and identification of Brucella organisms in Iran. Iran J Clin Infect Dis. 2008;4(3):185-8.

6. Karsen H, Tekin Koruk S, Duygu F, Yapici K, Kati M. Review of 17 cases of neurobrucellosis: clinical manifestations, diagnosis, and management. Arch Iran Med. 2012;15(8):491-4.

7. Delpy IP, Kaveh M. The occurrence of brucellosisin Iran. Facult Medic veterin J.1945;1:35-9.

8. Kaveh M. Brucellosis in general.Tehran (In Persian). Facult Medic veterin J.1952;5:17-30.

9. Haji-Abdolbagi M, Rasooli-Nejad M, Jafari S, Hasibi M, Soudbakhsh A. Clinical and laboratory findings in neurobrucellosis: review of 31 cases. Arch Iran Med. 2008;11(1):21-5.

10. Dean AS, Crump L, Greter H, Schelling E, Zinsstag J. Global burden of human brucellosis: a systematic review of disease frequency. PLoS Negl Trop Dis. 2012;6(10).

11. Young EJ. An overview of human brucellosis. Clin Infect Dis. 1995;21(2):283-9. quiz 290.

12. Sofian M, Aghakhani A, Velayati AA, Banifazl M, Eslamifar A, Ramezani A. Risk factors for human brucellosis in Iran: a case-control study. Int J Infect Dis. 2008;12(2):157-61.

13. Gul HC, Erdem H, Bek S. Overview of neurobrucellosis: a pooled 
analysis of 187 cases. Int J Infect Dis. 2009;13(6):e339-43.

14. Madkour MM, Al-Moutaery K, Al-Deeb S, Al-Swailem R, Al-Okaily F. Madkour's Brucellosis.:Springer;2001. Neurobrucellosis. pp.166-78.

15. Shoaei S, Nasri Razin B, Family A, Nabavi M. Neurobrucellosis; A Case Report. Arch Clin Infect Dis. 2007;2(4):203-5.

16. Rose BD, Post TW, Narins R. Clinical physiology of acid-base and electrolyte disorders. New York:McGraw-Hill;2001.

17. Baldi PC, Araj GF, Racaro GC, Wallach JC, Fossati CA. Detection of Antibodies to BrucellaCytoplasmic Proteins in the Cerebrospinal Fluid of Patients with Neurobrucellosis. Clin Diagn Lab Immunol. 1999;6(5):756-9.
18. Al-Sous MW, Bohlega S, Al-Kawi MZ, Alwatban J, McLean DR. Neurobrucellosis: clinical and neuroimaging correlation. AJNR Am J Neuroradiol. 2004;25(3):395-401.

19. Kochar DK, Agarwal N, Jain N, Sharma BV, Rastogi A, Meena CB. Clinical profile of neurobrucellosis-a report on 12 cases from Bikaner (north-west India). J Assoc Physicians India. 2000;48(4):376.

20. Turel O, Sanli K, Hatipoglu N, Aydogmus C, Hatipoglu H, Siraneci R. Acute meningoencephalitis due to Brucella: case report and review of neurobrucellosis in children. Turk J Pediatr. 2010;52(4):426-9. 